\title{
Health Outcomes for Better Information and Care (HOBIC): Integrating Patient Outcome Information into Nursing Undergraduate Curricula
}

Carole Orchard, RN, BSN, Med, EdD

Director \& Associate Professor, School of Nursing

University of Western Ontario

London, ON

Cheryl Reid-Haughian, RN, BHSCN, MHSC

Director, Professional Practice

ParaMed Home Health Care

Ottawa, ON

Rick Vanderlee, RN, BNSc, MScN, EdD

Dean, Arts \& Science and Acting Director, School of Nursing

Nipissing University

North Bay, ON

Currently, there is no standardized approach in practice for collecting and organizing data and information on health outcomes for most of our healthcare disciplines including nursing, pharmacy, occupational therapy and physiotherapy. For many years, nurse administrators have experienced frustration over the lack of significant data regarding nurses' work that has a direct impact on patient outcomes. This situation has been particularly problematic when justifying budgets or publicly demonstrating how nurses directly affect the health of their patients and clients. At the same time, given the range of personnel who deliver direct care to patients, collecting data related to patient outcomes would allow administrators to guide their allocation of care to other regulated and non-regulated healthcare workers. This significant shift in the use of patient outcomes in the practice setting needs to be integrated into nursing education curricula so that students will be prepared conceptually, technically and practically for the changes that are currently happening. 


\section{Integrating HOBIC Patient Outcomes into Nursing Undergraduate Curricula}

HOBIC builds upon work that began in 1999 as the Nursing and Health Outcomes Project (NHOP). An expert panel commissioned a critical appraisal of research to identify patient outcomes that are influenced by nursing (Doran 2003). A study to test the feasibility of collecting these outcomes in a systematic and standardized form across acute and chronic care hospitals, nursing homes and home care was undertaken across Ontario (Doran et al. 2006) and demonstrated that nurses were receptive to assessing these outcomes, could do it in a reliable and efficient way and valued the information produced. Based on these results, a recommendation to the Ontario Ministry of Health and Long-Term Care (MOHLTC) to implement the assessment and collection of these outcomes was accepted as the Ministry concluded that these outcomes are critical components in its strategy to monitor patient health outcomes. Thus, planning is well underway to support nursing personnel in a variety of settings who are learning to adopt the health outcomes in their practice. A key group within this strategy is nurse educators and their students - our future nursing professionals. Integration of health outcomes into nursing education programs supports nursing education's strong emphasis on the development of nursing professionals who see evidencedbased practice as a critical element in care delivery. This emphasis is further reinforced through the newly revised
Canadian Association of School of Nursing (CASN) accreditation standard for Knowledge-Based Practice, which states: "Learners have opportunities to engage in effective knowledgebased practice that is safe and ethical" and "Learners acquire and apply critical appraisal skills related to evidence from a variety of sources, including research throughout the program" (the standard's key element). The HOBIC Project and its patient outcomes provide a means for nursing education to meet this standard. A further advantage in adopting patient outcome assessments in nursing education programs is their capacity to reflect nursing regulators' documentation standards. An example is the Nursing Documentation Standard of the College of Nurses of Ontario (CNO): "[A] nurse maintains documentation that is: a complete record of nursing care provided including: assessments, identification of health issues; a plan of care; implementation; and evaluation" and the evidence suggested to meet the same: (a) "documenting an assessment of the client's health status and situation/ circumstances" and (b) "documenting an evaluation of nursing strategies and client outcomes" (CNO 2002).

Incorporating nursing-sensitive outcomes into existing curricula will help students make the connection between patient assessments, nursing interventions and patient outcomes and reinforce the value of evaluating their patient care delivery. The concern that nurse educators will likely express is where - in the already crammed curriculum - can yet another 
component be added? We would like to propose not an addition, but a complementary way of preparing students.

We propose introducing the HOBIC patient outcomes as part of nursing students' health assessment and therapeutic communication development and in their informatics classes on electronic patient charting. The HOBIC project is developing a computerized set of forms for several settings: (a) acute care, (b) complex continuing care, (c) long-term care and (d) home care. Access to this programming through nursing education programs will facilitate students' computer entry of assessed data in laboratory settings and prepare them for assessing HOBIC patient outcomes in the health agencies where they gain practical experience. These health outcomes are grouped as follows: activities of daily living (ADL), bladder continence, pain symptoms, fatigue, dyspnea, nausea, falls, pressure ulcers and, finally, therapeutic selfcare. Nursing practice labs (especially clinical simulation labs) focusing on these areas could integrate the data collection components from HOBIC assessment forms as the means to guide students' documentation of "patient" assessments during classes. Faculty who teach and supervise students in clinical settings could then employ the appropriate HOBIC form to guide students' documentation of assessment and care of their patients. The agency need not necessarily employ the HOBIC measures as part of its charting norms. As students progress in their nursing programs, more emphasis can be placed on reflection about the outcomes of their nursing interventions, using the HOBIC forms as a guide. Students who become familiar with the HOBIC patient outcomes will be able to guide their preceptors and other nursing staff in the application of these outcomes as the students progress through their nursing programs and practice settings. The HOBIC approach will become their norm of practice upon graduation and entry into professional nursing practice.

Another approach to integrating HOBIC outcomes into nursing education is through simulated labs. In Ontario, MOHLTC funded development of simulated labs for all nursing schools in the province. These labs now have the capability to program mannequins that can respond to questions from students about a range of health problems. Creative case scenario development could include all the components of the HOBIC patient outcomes as a means for students' data collection during assessment of these "clients," and also facilitate documentation of students' findings into electronic patient records. Furthermore, case studies could take the "patient" through discharge, stimulating students to evaluate client self-care and preparation of the client for discharge to another level of care. Subsequently, use of HOBIC patient outcomes in simulated or role-played situations could help students link empirical evidence from the lab-based scenarios with their nursing interventions and stimulate them to speculate on actual outcomes (Doran 2003). 


\section{Orientation to HOBIC Patient Outcomes}

An online and a CD-based orientation to the use of the HOBIC patient outcomes are now being developed and will be available for testing this fall. All nursing programs in Ontario are expected to have access to this program; as the adoption of HOBIC expands, these tools will be further accessible. The HOBIC team members decided to move from a traditional behaviourist "train-the-trainer" model of implementation to discovery- and inquiry-based learning, a model now widely used in nursing education. The primary focus for this strategy is change management. Several principles of change management laid the foundation for the strategy: (a) ensure that nurse educators are involved in the change process; (b) understand nurse educators' environmental readiness for the change; (c) tailor the learning program for nursing students;

(d) identify nurse educator leaders, both formal and informal, who have the capability of sustaining the change; (e) be strategic: determine how to integrate change into the nursing education programs' vision; (f) link the change to best practices in patient care; $(\mathrm{g})$ provide evidence that the proposed change works; (h) dedicate resources to the implementation process; and (i) understand that all change is an evolutionary process and takes time to adopt. A parallel approach is to be developed for service providers.

The learning and development strategy reflects the standardized measures of functions, symptoms and outcomes adopted into databases. While the tools are fixed, the learning strategy must accommodate a blended approach. To meet the needs of diverse groups of nurses, the blend might include learner facilitators in service provider groups, self-directed approaches provided via Web- or CD-based interactive programs, or paper-based materials. Learning will focus around realistic case studies, with knowledge developing as the learner moves through each study. The cases will include the patients' perspective about their symptoms, experiences and feelings. The goal of the learning is to orient nursing students to focus on outcomes in their care of patients and clients. Self-assessment segments will help learners evaluate their knowledge, with help-points to direct them back to appropriate sections as necessary.

The HOBIC educational program is designed to help nursing students understand patient-sensitive outcomes and how to assess and document their evaluation. With due acknowledgement of nursing students' workload and their limited access to electronic data systems, the learning will be structured around case studies of realistic patient situations to help them gain knowledge of ratings for each outcome. As nursing students begin to apply nursing-sensitive patient outcomes in their practice settings, nursing faculty coordinators will assist with face-to-face sessions that will augment the standardized learning and ensure that implementation reflects the unique features of the agencies where students gain their clinical practice. 


\section{Summary}

Nursing-sensitive outcomes provide common information across sectors, thus eliminating duplication that frequently occurs as individuals move across settings. These outcomes also facilitate increased trust among colleagues and support common understandings of patient care needs, thus enhancing continuity of care. Outcomes-oriented information is also likely to increase patient safety and improve overall quality of care. Shared standards and data support consistent decision-making, as nursing decisions can be tracked back over time to assess patient care outcomes. Consequently, nurses will have the means to determine the impact of their interventions on patient outcomes.

At the same time, adoption of common approaches to patient assessment leads to greater professional accountability and moves nursing care from a task orientation to an outcomes focus. For administrators, such improvements in monitoring and evaluating patient outcomes translate into improvements in efficiencies and effectiveness, thus providing a return on investment in implementing these outcomes within their agency. For nurses, integration and utilization of outcomes information increases the visibility and significance of their decision-making and patient care. Together with patients, nurses can utilize the outcomes information to make evidence-based decisions and advocate for appropriate care. At an aggregate level, the use of outcomes information creates a continuous feedback loop that is essential to ensuring evidencebased care and the best possible patient outcomes, not only for individuals, but also for families, communities and populations.

Outcomes-oriented care provides a gateway for transforming the way we care for patients; puts safe, ethical, high-quality care for patients first; embodies the principles of evidencebased practice; ensures that the value of nursing is clearly understood within the larger system; and ensures that the requirements for measurability and accountability can be achieved. This journey is continuous and is being expanded to engage all other health disciplines in understanding and documenting their contributions to patient care, both as individual practitioners and as members of a healthcare team. Preparing nursing students in an outcomes approach will facilitate systemwide adoption of HOBIC patient outcomes over time and provide a means to determine the impact of nursing care on our patients.

\section{Acknowledgements}

The authors acknowledge their colleagues in the HOBIC Learning and Development Committee whose shared work helped produce this paper: Rivie Seaberg (chair), Julia Scott, Carl Gray, Judith Horrigan, Karen Fleming, Nancy Curtin Telegdi, Rosemary Watkins, Karen Serediuk, Joe Racz, Ginnuy Odette, Dr. Dorothy Pringle and Peggy White. 


\section{References}

Canadian Association of Schools of Nursing (CASN). 2005. Manual for the CASN

Accreditation Program. Ottawa: Author.

College of Nurses of Ontario (CNO). 2002.

Nursing Documentation Standards (rev. ed.).

Toronto: Author.

Doran, D.M., ed. 2003. Nursing-Sensitive

Outcomes: State of the Science. Toronto: Jones \& Bartlett.

Doran, D.M., M.B. Harrison, H.S. Laschinger, J.P. Hirdes, E. Rukholm, S. Sidani, L. McGillis-

Hall and A.E. Tourangeau. 2006. "Nursing-
Sensitive Outcomes Data Collection in Acute Care and Long-term Care Settings." Nursing Research 55(2 supplement), 875-81.

Health Results Team for Information Management (HRT-IM). 2005 (August). Information Management News Issue \#3.

McGillis Hall, L., ed. 2004. Quality Work Environments for Nurse and Patient Safety. Toronto: Jones \& Bartlett.

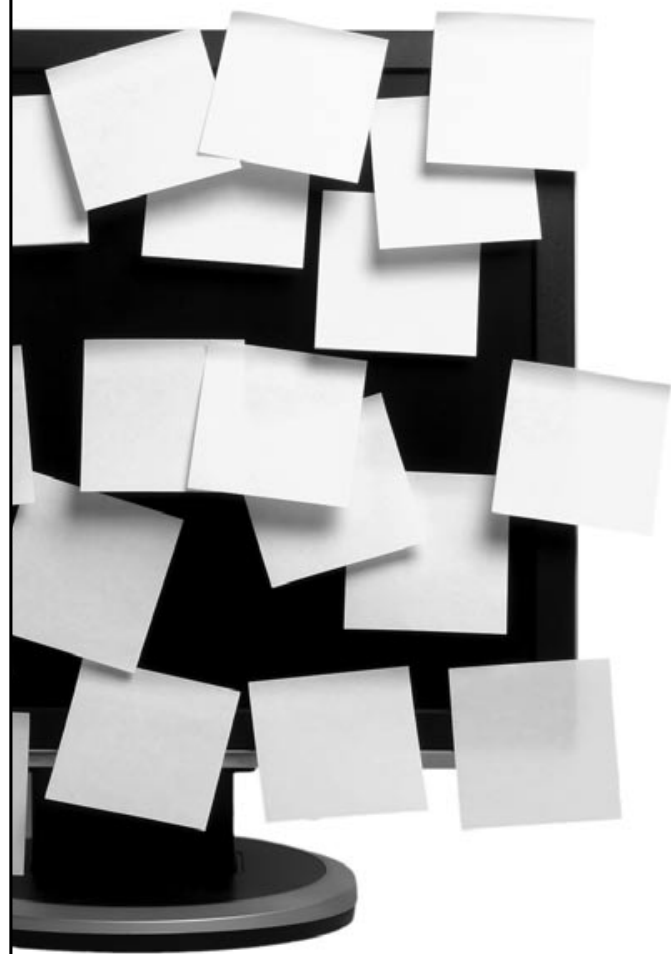

\section{Get it first. Get it fast.}

Sign up to have the table of contents e-mailed to your inbox each time a new issue comes online.

The table of contents allows you to:

- Scan the contents of each Longwoods journal

- Prioritize your reading instantly

- Link directly to articles that are of interest to you

www.longwoods.com 